\title{
Force transducers - A review of design and metrological issues
}

\author{
Rajesh Kumar ${ }^{a^{*}}$ and S. Maji ${ }^{b}$
}

${ }^{a}$ CSIR-National Physical Laboratory, New Delhi, India ${ }^{b}$ Delhi Technological University, Delhi, India

\begin{tabular}{l}
\hline A R T I C L E I N F O \\
\hline Article history: \\
Received 6 September, 2015 \\
Accepted 4 December 2015 \\
Available online \\
4 December 2015 \\
\hline Keywords: \\
Force transducer \\
Uncertainty of measurement \\
MEMS \\
Strain gauge \\
\hline
\end{tabular}

\section{A B S T R A C T}

The present work reports the retrospective investigation of force transducers widely used for force measurement related applications in variety of areas. The paper discusses the salient features of different types of force transducers along with their design and metrological features. The force transducers are covered from the analogue type to modern force transducers. The paper attempts to discuss the limitations of the different types of force transducers.

MEMS

\section{Introduction}

Force transducers have been used over the decades for measurement of force and to maintain traceability of force from force standard machines to the functional areas where it is required. The force transducers are used in various engineering applications like electronic weighing scales, verification of material testing machines, measurement of cutting forces in various machining processes and thrust force measurement in rockets etc. The present form of force transducers has been gone through various transformations since their inception i.e. ancient era (Marlowe, 1975). The force transducers may be broadly categorized into analogue type and digital type. The analog type mainly includes strain gauged force transducers. Some other force transducers may be like tuning fork type and MEMS force transducers.

\subsection{Dial Gauged Force Transducers}

The dial gauged force transducers are essentially a sensing element, equipped with a dial gauge to measure the deflection under the action of external forces. The deflection is a measure of the force

\footnotetext{
* Corresponding author.

E-mail addresses: kumarr@nplindia.org (R. Kumar) 
applied. Dial gauged force transducers are still used over large scale for applications with coarser measurement capabilities. The dial gauged force transducer offers simple design and manufacturing considerations. At the same time, dial gauge suffers shortcomings of being mechanical system and poor resolution. The dial gauge provides though convenient but coarser mean of deflection measurement (Kumar and Kumar, 2011).

\subsection{Strain Gauged Force Transducer}

Strain gauged force transducers are developed in late 1960's and offers very reliable force measurement. Strain gauge force transducers offers measurement of force in terms of electrical form like voltage as output of the Wheatstone bridge, according to which the strain gauges are arranged. Strain gauged force transducers are most widely used various force measurement applications precisely. The strain gauged force transducers offers improved uncertainty of measurement with accurate and reliable measurement capability.

\subsection{Tuning Fork Type Force Transducers}

Tuning fork type force transducers have supposed to be potentially better long-term stability than conventional strain gauge types as they directly convert applied force to resonant frequency. Such force transducers are currently developed for some nominal capacity and have been experimented to serve as force transfer standard. National Institute of Metrology, Japan (NMIJ) has designed, developed and demonstrated a new force transducer of $50 \mathrm{~N}$ rated capacity which adopts a double-ended tuning fork (DETF) sensing unit. The performance of DETF force transducers was evaluated using the $500 \mathrm{~N}$ force standard machine and they are found to be better than strain gauged force transducer. Such DETF force transducers are found to have low creep, low hysteresis, low temperature coefficient and superior longterm stability and are suitable to serve as force transfer standards like strain gauged force transducer.

\section{Present Status of Force Transducers}

Dhawan et al. (2004) have discussed some of preliminary results on the design and fabrication of a $5 \mathrm{kN}$ force gauge and its metrological characterization as per the calibration procedure based on documented standards. The force transducers so developed are found to have relative deviation due to repeatability better than $\pm 0.002 \%(k=2)$ and the overall estimated uncertainty in the force measured is better than $\pm 0.05 \%(k=2)$ when combined with the reproducibility and the interpolation. The results so obtained compare favorably among themselves irrespective of the force gauge fabricated in one batch or in different batches.

Titus et al. (2009) describes some preliminary results of the design, development and characterization of precision strain gauged artifacts for the measurement of low forces of $1 \mathrm{~N}$ and $2 \mathrm{~N}$, which has been a vital field for various applications like micro hardness and to link the newton range to sub - newton range of force. Stress - strain distribution has been obtained for designed shape by finite element analysis and the location of the strain gauges has been defined according to the stress strain pattern over the surface of the element. For optimum results, the strain gauges are applied over the locations to provide most sensitivity and are fixed to surface with high quality epoxy adhesive. 50 $\mathrm{N}$ dead weight force machine with uncertainty of force realization $0.0012 \%(k=2)$ has been used for characterization of these artifacts. The relative uncertainty due to repeatability and reproducibility of the developed artifact are found up to $0.003 \%(k=2)$ and $0.006 \%(k=2)$ respectively (Titus et.al, 2009). Chen et al. (2007) discussed the stress and strain investigations in a ring strain sensor, subjected a compressive force at its upper and lower top points using finite element analysis as a tool. The ring shaped force transducers are commonly used through the globe due to simplicity in manufacturing and design considerations for precision force measurement related applications. The stress - strain

distributions implicates the locations of stress - strain during metrological investigations of the force 
transducer. The stress - strain computed by computational means is being compared by measuring the stress - strain by experimental means. The stress - strain is measured by applying the strain gauges at suitable locations by arranging the strain gauges according to Wheatstone bridge. A correlation has been obtained to indicate the coherence of experimental and computational measurement of stress strain. The study further discusses alternative locations for application of strain gauges in case of ring shaped force transducer.

Yaldiz et al. (2007) have studied that the cutting forces generated in metal cutting process have a direct influence on generation heat, tool wear or failure, quality of machined surface and accuracy of the work piece during the machining process like milling, drilling and turning. In this study, a milling dynamometer that can measure static and dynamic cutting forces and torque during the machining process has been designed and developed. An octagonal ring type cutting force dynamometer has been fabricated and the orientation of octagonal rings and strain gauge locations has been determined to optimum sensitivity. A suitable data acquisition system has been used to extract the signals and processing of data. An extensive investigation of the cutting force dynamometer reveals that the dynamometer is reliable enough to be used to measure static and dynamic cutting forces and torque.

Kim et al. (2003) studied that a sensing element of column type has been designed as a multicomponent force / moment sensor by attaching strain gages for force measurement. The different design aspects of the force transducer like the ratio of length over diameter (L/D) for the sensing element was designed analytically and validated by computational tools like finite element analysis. The procedure adopted and the results are discussed related to the column type force transducer.

Stefanecsu et al. (2003) also demonstrated that a strain gauge transducer that can alternatively measure force or pressure depending upon the requirement and application. Functional aspects of the transducers have been discussed with suitable description. Computer Aided Design and other modern tools are employed by the researcher in determining the optimal geometry of the elastic element along with finite element analysis, ensuring flexible and interactive modifications of the geometry and the correlation with the manufacturing process.

Stefanecsu and coworkers (2006) further showed that only few types of flexible structures are recommended for measuring very large forces which is very important area for heavy industries and high capacity presses. Author stresses upon the axi-symmetrical shapes for design and development of strain gauged force transducers and correlates the ease of application of strain gauges in such shapes. Numerical investigations on various models of $\mathrm{N}$ - shaped strain gauged elastic elements are presented together with their advantages in the field of precision force measurements (Stefanecsu et al., 2006). In other research work, Stefanecsu et al. (2007) showed correlation among Force, Mass, Pressure, Acceleration, Vibration and other mechanical quantities by their physical formulas and their similar measurement principles. Force is a derived unit of mass with time and length. Despite of many methods available for force measurement, strain gauged force transducers are predominant.

Joo et al. (2002) discussed the paper describing the design process and evaluation results of a compact six-component load cell, which is area of recent development and related to multi component force / torque transducer in addition to uni-axial force / torque transducers. A new six-component load cell including a ring-type structure as the basic sensing element has been developed. The location of strain gauges applications have been identified using finite element analysis and arranged accordingly into Wheatstone bridge (Joo et al., 2002).

Field and Beams (2005) studied the project report that discusses the basics of strain gauges and how to use strain gauges for load cell development for measurement of force. The report discuses fundamental theory of the strain gauges, their constructional details and principle. The strain gauges are arranged in Wheatstone bridge as each strain gauge represents the arm of Whetstone bridge. The 
change in resistance is measured, when the force is applied to the force transducer. The fundamentals of strain gauged force transducer have been discussed.

Robinson (1997) showed that the mechanical interfaces, such as bolted joints, within load cell element should be prevented as they can cause considerably high hysteresis. Relevance of aspect ratio has been discussed in the paper and it is shown that significant hysteresis may result from radial slip at the interface between the load cell and its loading pads. Circular cylindrical load cell elements have been modeled and analyzed by finite element analysis. Contact stresses were computed for ascending and descending series of calibration forces to calculate contact hysteresis. The finite element findings are found in coherence with the experimental findings.

Andrae et al. (2003) discussed the evaluation of the uncertainty of forces realized with force standard and force calibration machines requires precision force transfer standards for inter-laboratory comparisons with minimum measurement uncertainties even with changing mounting conditions during the calibration procedure adopted according to standard procedure. A new transfer force transducer has been discussed, having reduced mounting-position-dependent measurement uncertainty due to its mechanical and structural features. Method for improved uncertainty has been discussed.

Tegtmeier (2005) studied that the structural surveillance requires the precision and continuous measurement of states of stresses / forces for measurement related purposes. An enclosed sensor has been developed to protect strain gauging of the sensing element of the force transducer. Suitable mechanical related fixtures are prepared and sensor is fabricated. Strain gauges are placed under suitable protective covering. The protective covering is helpful in improving life span of the force transducer. Park and Kang (2005) described the various uses of a build-up system, which have been used for higher capacity force measurement. It can be used with a large-force standard machine in the range of $1 \mathrm{MN}$ or higher. A force standard machine of $10 \mathrm{MN}$ capacity at Korea Research Institute of Standards and Science (KRISS) along with the calibration of a high capacity material-testing machine is described. Finally, an evaluation method for a deadweight force machine is described as an example of such higher range build up systems (Park \& Kang, 2005).

Fank and Demirkol (2006) studied the application of heat treatments on elastic elements of the transducers. It has been a very effective method for attaining good performance in force measurements for calibration and other applications. Heat treatments change the microstructure of the material of the sensing element, whose characteristics are deciding factor for performance of force transducers majorly relative uncertainty due to hysteresis. The present study investigates the changing microstructure of AISI 4340 steel by the application of different heat treatment processes, and the subsequent measurement of the hysteresis performance of force transducers in relation to the change in the structural characteristics of the spring elements. The results indicated that the hysteresis characteristics of force transducers with quenched and tempered materials have been improved with increasing hardness.

In 2005, Pusa made a study during past 20 years that has shown it is better to evaluate and summarize the performance of force transfer standards to evaluate the force standard machines in comparison to other methods. The study includes and extensive investigations of the force transfer standards for past 20 years, to summarize their values at different nominal forces, critical analysis of deviation if any. The study points out that it is very important to have ultra precision force transducers as force transfer standards due to superior metrological characteristics. Author suggests the following key points to affirm the metrological capability of force standard machines (Pusa, 2005):

- $\quad$ selecting the transducers according to the technical information

- $\quad$ fixing the measurement program and use it systematically 
- $\quad$ hold the transducers in life with supply voltage and periodical loadings,

- $\quad$ take care of the mechanical behavior, loading parts and mechanical handling of the transducers.

If possible, the transfer transducers should have an age more than five years, they should be used regularly and the characterization data must be known and updated. Schwind and Hahn (2009) described the analysis and evaluation of calibration of several precision force transducer types, each transducer by a precision amplifier at $5 \mathrm{~V}$ and $225 \mathrm{HZ}$ carrier frequency excitation voltage and by a precision amplifier at $5 \mathrm{~V}$ direct current excitation voltage. Calibration procedure is adopted according to standard ISO 376 to determine the uncertainty. Investigations are made to study the influence of the excitation voltage over the metrological characteristics of the force transducers with goal to see the scope of interchangeability of different amplifiers (Schwind \& Hahn, 2009). Pal et al. (2010) emphasized on the establishment of traceability for the strain measuring data acquisition system in terms of voltage for force transducers. It is utmost to maintain the chain of traceability. In case, amplifier's output voltage is not calibrated then traceability chain breaks. To maintain the traceability chain, the amplifier's output voltage has been calibrated for corresponding strain in terms of voltage. The sensitivity is calculated using calibration results and further used to feed in data acquisition system to display the result in terms of force / strain.

Yee (1992) has given information regarding the automation of the dead weight force machines of different capacity ranging from $2.2 \mathrm{kN}$ to $4.45 \mathrm{MN} \&$ automating the measurement using strain gauge load cells at National Institute of Standards and Technology (NIST), USA. An in-house computer program has been developed to control / operate the force machines and generation of calibration report (Yee, 1992). Stoian et al. (2007) discussed a new type of force sensor. The sensor consists of two spring discs, positioned in mirror one to another and joined together. Sensing element is placed between the spring discs. The sensor geometry has been designed using finite element analysis (FEA), applied to simulate the mechanical strains and stresses and to improve the sensor characteristics. Calibration performed using a testing machine with dead weights to evaluate the linearity, hysteresis and other characteristics (Stoian et al., 2007). The ring shaped force transducers are modified to different shapes like square, hexagonal, octagonal and extended octagonal ring etc. for different applications. The static force measurement related applications are very important including material testing machines, thrust measurement, electronic weighing scales etc. like dynamic force measurement related application like measurement of cutting forces.

There has been little attention has been paid to the development of simple shaped force transducers for static force measurement, although commercially manufactured force transducers are available. The ring shaped force transducers have ease of design as well as manufacturing. The force transducer is developed on the basis of thin rings and has been analysed by analytical method as well as computational method. The computational investigations are very helpful in identifying suitable locations of strain gauges over the force transducers (Kumar \& Sharma, 2012). The Institute of Measurement and Control's report reviews the performance and applications of strain gauged load cells that output their measuring signal in a digital format as opposed to conventional analog signals. The performance of load cells is compared to conventional analogue load cells for industrial process weighing applications. This monograph reviews all of the features relating to strain gauged load cells that incorporate data acquisition to provide measuring output signals in a digit form (Institute of the Measurement and Control, 2003). ISO 376 (2011) is the most widely accepted standard procedure for calibration of force transducers and discusses the different factors pertaining to evaluation of uncertainty of measurement of force transducers to different classes depending upon the individual uncertainty component as well as overall uncertainty of measurement of the force transducer. Recently, in India, ISO 376 (2011) has been adopted and the standard IS 4169: 1988 (reaffirmed 2003) has been renamed as IS 4169: 1988 and is according to the guidelines of ISO 376: 2011 (ISO 376, 2011 and IS 4169, 2003). 


\section{Design and Development of Indigenous Strain Gauged Force Transducer}

In the present paper the design and simulation of a force transducer made up of EN 24 steel for the measurement of medium forces up to $5 \mathrm{kN}$ has been presented. The force sensors for medium range of forces are quite useful for Indian industry. In this resistance strain gauges are used for the extraction of electrical signal and the gauge factor of the strain gauge is 2.1. The chosen EN 24 steel diaphragm for the design of force transducer is shown in Fig. 1, the diaphragm size is $120 \mathrm{~mm}$ in diameter and its thickness is $3 \mathrm{~mm}$. The maximum stressed areas have been determined for the optimal placement of strain gauges to get the highest sensitivity.

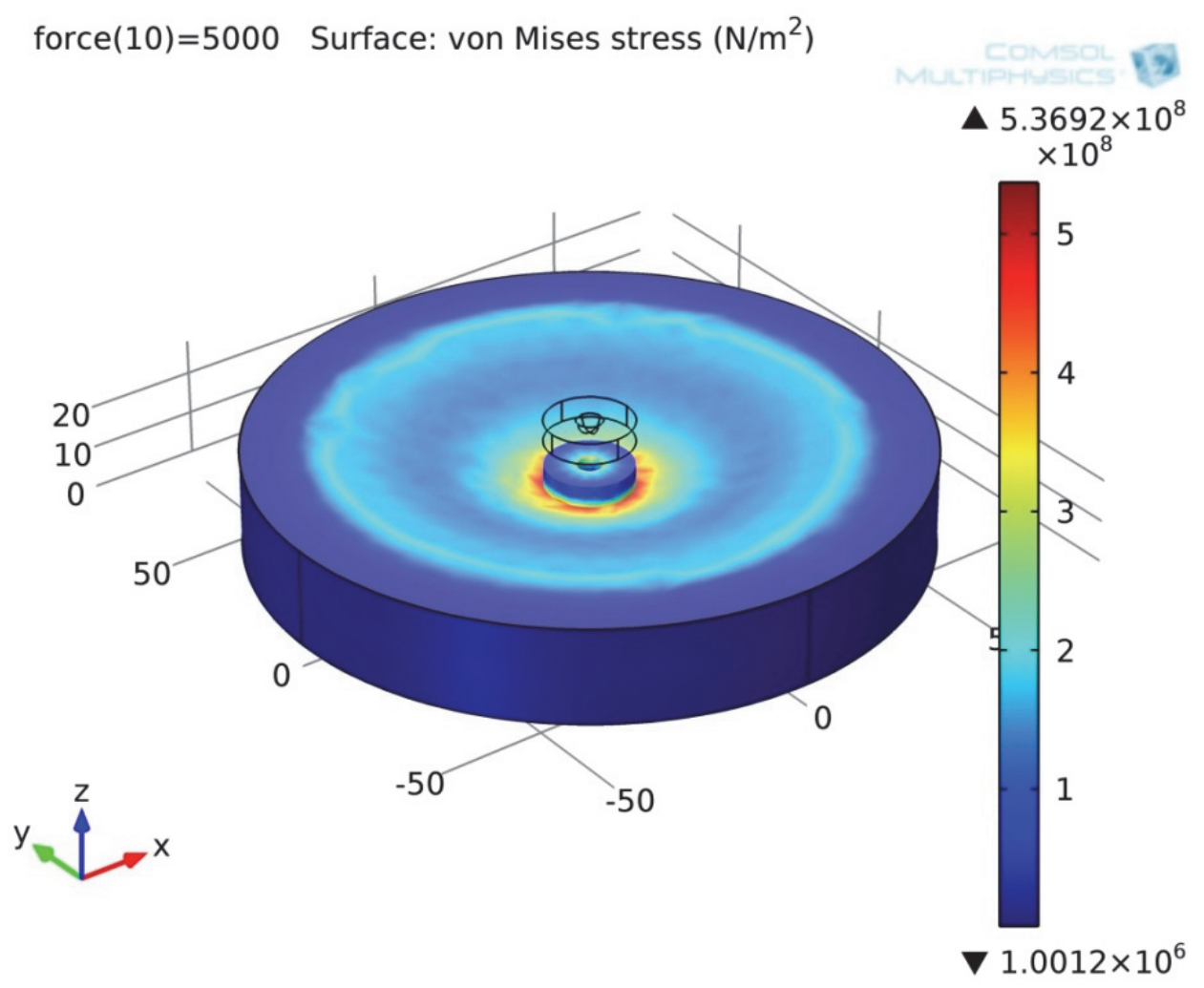

Fig. 1. Stress of the diaphragm at $5000 \mathrm{~N}$ force

Based on the findings of the finite element analysis, the strain gauges are located at an angle of $90^{\circ}, 180^{\circ}, 270^{\circ}$ and $360^{\circ}$ from vertical axis on the outer surface of the transducer as the surface is made flat. Strain gauges arranged as per the Wheatstone bridge configuration to nullify the temperature effect. As the external force is applied, the bridge gets unbalanced and the resultant output in form of $\mathrm{mV} / \mathrm{V}$ (an electrical form) represents the force applied. The force transducer has been calibrated according to the calibration procedures using the $50 \mathrm{kN}$ dead weight force machines as discussed earlier. The dead weight force machine has a hanger (of nominal force $0.5 \mathrm{kN}$ ) with stack to which all weights are attached (Kumar and Kumar 2011). The dead weight force machine is able to apply the force in ascending or descending order according to the calibration procedures based on standards ISO 3762011 as well as IS 4169-1988 (reaffirmed 2003). A very high resolution digital indicator (DK 38 of HBM, Germany) of resolution $0.00001 \mathrm{mV} / \mathrm{V}$ has been used for taking the observations.

The relative combined standard uncertainty $w_{\mathrm{c}}$ (tra) and the relative expanded uncertainty $W_{\text {(tra) }}$ for $k=2$ will be calculated by the following Eqs. (1-2). Other terms represents the relative variances of relative uncertainty due to repeatability (rep), reproducibility (rpr), hysteresis (hys), zero (zer), interpolation (int) and resolution (res). 


$$
\begin{aligned}
& w_{\mathrm{c}(\text { tra) }}=\left[w_{\text {rep }}^{2}+w_{\text {rpr }}^{2}+w_{\text {int }}^{2}+w_{\text {hys }}^{2}+w_{\text {zer }}^{2}+w_{\text {res }}^{2}\right]^{1 / 2} \\
& W_{\text {(tra) }}=k \cdot w_{\mathrm{c} \text { (tra) }}
\end{aligned}
$$

The relative uncertainty of calibration $W$ shall be determined by the Eq. (3), considering the best measurement capability (bmc) or uncertainty of force measurement by force machine of the force standard machine.

$$
W=\left[W_{(\text {tra })}^{2}+W_{(\text {bmc })}^{2}\right]^{1 / 2}
$$

Uncertainty of measurement is found up to $0.05 \%(k=2)$, while taking into the necessary factors into considerations.

\section{In-house Investigations of MEMS based Force Transducers}

A new class of force transducers has come up during the last one and half decade. This kind of load cells utilize the mechanical properties of single crystal silicon as load or force sensing element and piezoresistive properties of monocrystalline or that of polycrystalline $\mathrm{Si}$ for the transduction of the stress generated during the sensing of the load or force. The biggest advantage of this kind of load cells is that the Gauge Factor of the Si piezoresistors is very high (almost a couple of hundred times of that of the metallic strain gauges) leading to very high sensitivity (Mäuselei et al. 2008, 2009). Disadvantages are the high cost and lower fracture strength of the material. These limitations could be overcome through ingenious design, custom packaging and batch fabrication of the sensor. With the evolution of Micro Electro Mechanical Systems (MEMS) technology, Si load cells can be scaled down and batch fabricated with custom designs. Silicon load cells have extremely significant applications in biomedical and industrial segments requiring precise and reliable force measurements. Several kinds of Si load cells based on MEMS technology have been developed while a lot more to come for a variety of application segments (Wise 2007).

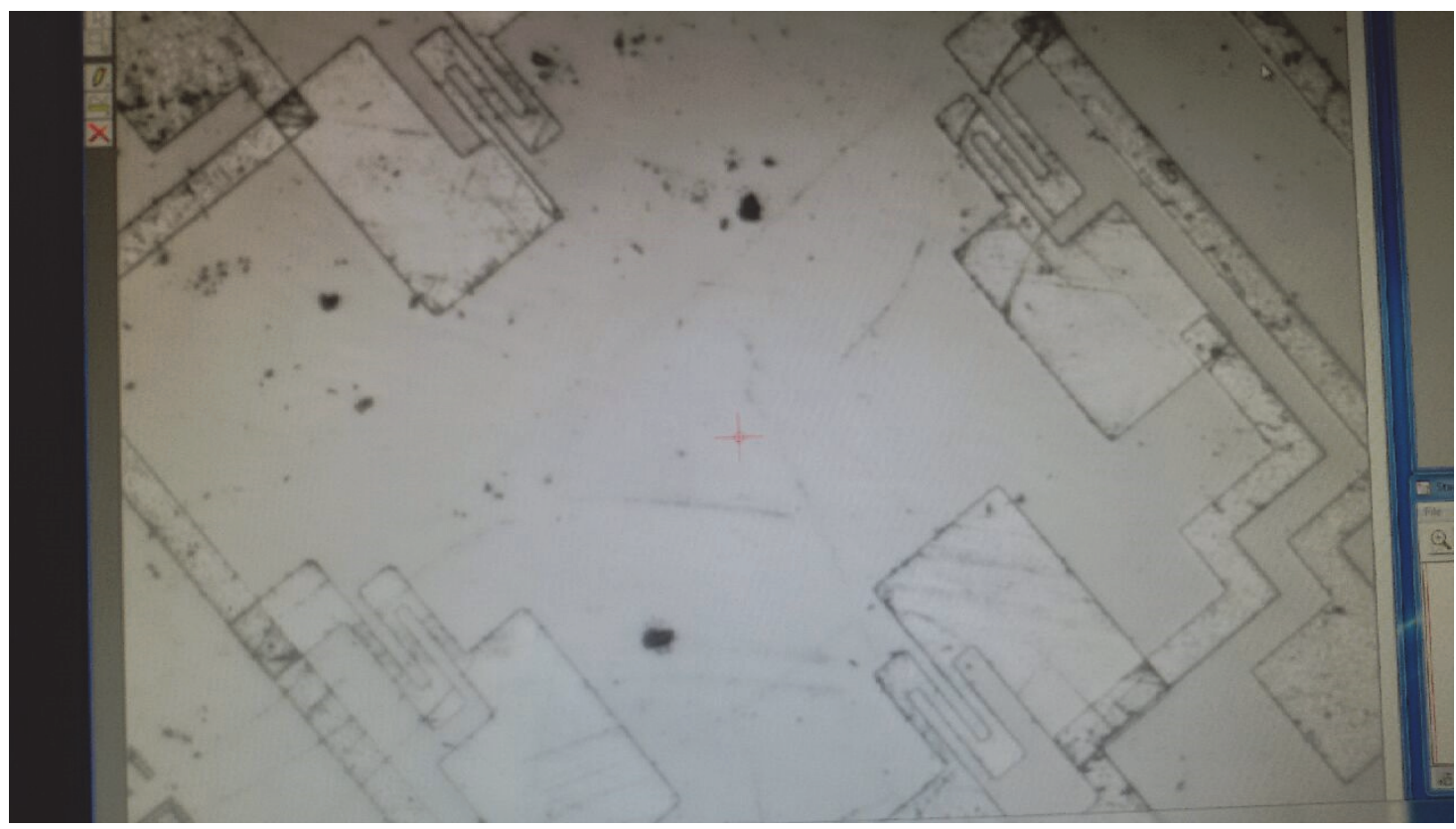

Fig. 2. A schematic view of MEMS Force Transducer.

Some MEMS based force transducers have been designed and developed in-house and their characterization is underway (Fig. 2). 


\section{Conclusion}

The review article presented attempts to discuss the brief of past research done related to the development and metrological issues of force transducers. The study involves review of various technical articles / report or monograph discussing issues like development of force transducers for different purposes, finite element computations, metrological performance etc. The review article reports that though force transducers might have been developed and used over the decades, a rationalized procedure for design and metrological related issues is still awaited. The force transducers developed are either developed only for some specific purposes or a particular type of design is discusses without discussing their significance and fundamental cause, why? Hence, this review report attempts to point out the lacks or shortcomings of past research so that a rationalized procedure for design and metrological issues of force transducers may be developed. To fulfill the raised objectives, an in-depth analysis is needed for developing a rationalized methodology for development of force transducers using conventional theories available, validation using computational tools like finite element analysis and metrological performance evaluation of force transducers thus developed using different standard procedures.

The work further reports in-house development of strain gauged force transducers. The strain gauged force transducers are designed and developed. Computational studies are made according to

Multiphysics $^{\mathrm{TM}}$, a software for finite element analysis. Strain gauges are applied over the suitable locations. Force transducer is calibrated according to standard calibration procedure ISO 376-2011 and uncertainty of measurement is up to $0.05 \%$, comprising of factors like relative repeatability, reproducibility, hysteresis, resolution etc. Further, in-house attempts are done to fabricate some MEMS based force sensors. The force sensors are fabricated and their characterization is underway.

\section{Acknowledgement}

Authors are thankful to Director, CSIR - National Physical Laboratory, New Delhi, India for his support to carry out the present research. 


\section{References}

Andrae, J., Hons, C., \& Sawla, A. (2003). High precision force transducer with new method for online compensation of parasitic effects. Measurement, 33(2), 173-178.

Chen, B., Wu, X., \& Peng, X. (2007). Finite element analysis of ring strain sensor. Sensors and Actuators A: Physical, 139(1), 66-69.

Dhawan, J. K, Jain, K. K, Titus, S. S. K \& Kumar, R., (2004). Design, development and characterization of $5 \mathrm{kN}$ force gauge. MAPAN-Journal of Metrology Society of India, 19(1-2) 61-67.

Fank, S., \& Demirkol, M. (2006). Effect of microstructure on the hysteresis performance of force transducers using AISI 4340 steel spring material. Sensors and Actuators A: Physical, 126(1), 2532.

Field, C. \& Beams, D. M, (2005). Project - GUISE, Introduction to strain gauges, Department of Electrical Engineering, University of Texas at Tyler, 1-7.

ISO, E. 376 (2011). Metallic materials. Calibration of force-proving instruments used for the verification of uniaxial testing machines (ISO 376: 2011).

IS 4169 (2014). Metallic materials - Calibration of force proving instruments used for verification of uniaxial testing machines (Bureau of Indian Standards, New Delhi, India).

Joo, J. W., Na, K. S., \& Kang, D. I. (2002). Design and evaluation of a six-component load cell. Measurement, 32(2), 125-133.

Wise, K. D. (2007). Integrated sensors, MEMS, and microsystems: Reflections on a fantastic voyage. Sensors and Actuators A: Physical, 136(1), 39-50.

Kim, J. H., Kang, D. I., Shin, H. H., \& Park, Y. K. (2003). Design and analysis of a column type multicomponent force/moment sensor. Measurement, 33(3), 213-219.

Kumar, H., \& Kumar, A. (2011). Investigations on measurement uncertainty and long term stability of dial gauged force proving instruments, NCSL International Measure Journal, 6, 64-68.

Kumar, H., \& Sharma, C. (2012). Role of finite element analysis in improving hysteresis error of force transducers. Transactions of the Institute of Measurement and Control, 34 (8), 1019-1024.

Marlowe D. E., (1975). A study of the national force measurement system, National Bureau of Standards, USA.

Mäuselein, S., Mack, O., Schwartz, R., \& Jäger, G. (2009). Investigations of new silicon load cells with thin-film strain gauges. In XIX IMEKO World Congress, Fundamental and Applied Metrology.

Mäuselein, S., Mack, O., Schwartz, R., \& Jäger, G. (2008). Single crystalline sensors with thin-film strain gauges for force measurement and weighing technology, Proc. of International Conference on Precision Measurement, Ilmenau.

Pal, B., Kumar, A., Madan, S., Ahmad, S., \& Govil, A. K. (2010). Establishment of traceability for strain measuring data acquisition system in terms of voltage. MAPAN-Journal of Metrology Society of India, 25(2), 125-128.

Park, Y. K \& Kang, D. I, (2005). Build-Up Force-Measuring System and its Applications, MAPANJournal of Metrology Society of India, 20(3), 223-229.

Pusa A, (2005). Long-Term Observation of Force Transfer Transducers as a Tool to Confirm the Measurement Capability of Force Standard Machines, MAPAN-Journal of Metrology Society of India, 20(3), 231-237.

Robinson, G. M. (1997). Finite element modelling of load cell hysteresis. Measurement, 20(2), 103107.

Schwind, D., \& Hahn, T. (2009). Investigation of the influence of carrier frequency of direct current voltage in force calibrations. In Proc. of XIX IMEKO World Congress (pp. 201-204).

Stefanescu, D. M., Dolga, L., \& Marinescu, A. (2003). Parametrical modeling of the strain gauged force and/or pressure transducer. Proc. of XVII IMEKO World Congress, Dubrovnik, Croatia.

Stefanescu, D. M. (2006). N-shaped axisymmetric elastic elements for strain gauged force transducers. Proc. of XVIII IMEKO World Congress, Rio de Janeiro, Brazil.

Ştefănescu, D. M. (2007). Strain gauged elastic elements for force and related quantities measurement. In CD Proc. IMEKO Int'l Conf. Cultivating Metrological Knowledge, Merida, Mexico, November (pp. 27-30). 
Stoian, A., Kuberczyk, T. \& Schultes, G., (2007). A new type of force sensor, IMEKO $20^{\text {th }}$ TC, $3^{\text {rd }}$ TC 16 and 1st TC22 International Conference, Merida, Mexico.

Tegtmeier, F., (2005). Microsensor for Stress Surveillance in Building Structures, MAPAN-Journal of Metrology Society of India, 20(3), 259-267.

Titus, S. S. K., Jain, K. K., Dhulkhed, S. K., \& Yadav, P. (2009). Desing and development of precision artifact for dissemination of low forces of $1 \mathrm{~N}$ and 2 N. Proc. of XIX IMEKO World Congress, Lisbon, Portugal.

Yaldız, S., Ünsaçar, F., Sağlam, H., \& Işık, H. (2007). Design, development and testing of a fourcomponent milling dynamometer for the measurement of cutting force and torque. Mechanical Systems and Signal Processing, 21(3), 1499-1511.

Yee, K. W. (1992, April). Automation of strain-gauge load-cell force calibration. In Proc. 1992 Natl. Conf. of Stand. Lab. Workshop and Symposium, Washington, DC (pp. 387-391). 